\title{
The Landscape of the Legal Aspects of Informed Consent for Medical Treatment in Papua New Guinea
}

\author{
Alfred P. Minei ${ }^{1}$, Rachelyn Ann S. Araña ${ }^{2}$, Ronald R. Roldan Jr. ${ }^{2}$ and Sam O. Kaipu ${ }^{1}$ \\ 1. School of Law, University of Papua New Guinea, Waigani, Post Office Box 126, National Capital District, Papua New Guinea \\ 2. Institute of Statistics, College of Arts and Sciences, University of the Philippines Los Baños, Los Baños, Laguna 4031, Philippines
}

\begin{abstract}
The major challenges militating against the proper practice of informed consent identified in our desk top review are related to cultural issues of the people. Many patients continue to value strongly their kinship ties with the past and believe in the traditional methods of healing sickness. The patients must be given some information about what the doctor proposes to do. The underlying spirit of informed consent is new in PNG, and it strives against the traditional attitudes of doctors that they know it best for the patients and the strong culture in customs and patients' misconceptions that affect their perceptions in making important health care decisions. Majority of the patients have some notion of informed consent, however not many people understand what it is. They still hang onto their traditional customs, beliefs and opinions that affect their health care decisions; they often look to their village elders, family and parents for advice in times of sickness or death in the family but where the law implies consent it is not often obtained. Summarization of current laws, reports of legal cases, and personal experiences were examined. Special requirements must be prescribed when patients are subjected to medical treatment. Documentation of a well-defined process, not only on paper, may not only protect the medical doctor from exposure to liability but increases the patient's autonomy in decisions concerning health and encourages compliance with treatment; and advances the interests of both patient and doctor. Lack of informed consent can reinforce a claim of medical malpractice, and could well undermine relevant health care policy to protect patient autonomy.
\end{abstract}

Key words: Autonomy, prior informed consent, valid consent, traditional custom.

\section{Introduction}

This review is based on an ongoing research on informed consent carried out in Papua New Guinea (PNG) at hospitals, district hospitals, health centers and private medical clinics. There are related publications which have been published in the previous issue of this journal and also in other publications.

Although this review would hope to identify other similar studies in this area in PNG which would provide a strong quantitative base of evidence for recommendations on informed consent, there is a lack of baseline information in PNG but other studies from other jurisdictions are cited although much of the quantitative literature on this topic is descriptive in nature.

Corresponding author: Alfred Minei, $\mathrm{PhD}$ candidate, research fields: law and medicine.
In this review we concentrate on patients attending healthcare institutions for medical treatment, the majority of who are illiterate and live in their local settings for many years. They adhere to their original cultural and religious values, beliefs and opinions. Their traditional customs are their sources of law and govern their daily activities. We focus this review on medical (or clinical) practice of informed consent. We discuss firstly what we know about informed consent, what it is, and the purposes it serves. Secondly, we discuss the issues that complicate the practice of informed consent. And finally, we make a few practical suggestions as to how healthcare professionals might optimally approach informed consent process in a developing country context.

This review makes sense with available knowledge on this subject, noting the empirical studies where possible, and summarizing relevant points that are particularly insightful. 


\section{Informed Consent}

Informed consent is the cornerstone of patients' rights. It is based on the inviolability of one person. It means the doctors do not have the right to touch or treat a patient without first obtaining the patient's approval because the patient is the one who must live with the consequences and deal with any discomfort caused by treatment. It is improper and even illegal if a medical doctor in a modern healthcare facility performs a treatment on a patient who does not agree to the treatment with full knowledge of what the treatment is, how the treatment is performed, and the major complications that the treatment could potentially cause.

In the treatment context, informed consent has a different purpose than in the research context. In treatment it requires a different level of protection and consent for the patient than for the research subject [1]. Because there is greater harm in research, there must be greater protection for a research subject [2]. We acknowledge that there is a rich literature on informed consent, so rather than providing a complete review of the doctrine of informed consent, this review only examined the doctrine in the treatment context from a developing country perspective.

The doctrine in the context of treatment has evolved through the medium of case law [3]. In other words, courts have addressed patients' claims that physicians failed to obtain proper authorization for the action they undertook [4]. Informed consent must be obtained before the patient receives treatment from a healthcare professional. It means a patient understands his or her condition and any proposed medical treatment. Patients have a legal right to be told any information that relates to their medical condition and treatment. Without this information, they are not able to make a fully informed choice and give valid consent for treatment. For example, in a clinical case, a young man was advised by his physician to undergo a laminectomy in an effort to alleviate back pain. The physician, aware that one percent of laminectomies resulted in paralysis, did not advise the patient of the risk because he believed this might cause the patient to reject the useful treatment. After the procedure, the patient fell from his hospital bed and was paralyzed [5]. It remained uncertain whether the laminectomy procedure or the patient's fall caused the paralysis. The doctor has a duty to explain the patient's medical condition, the recommended treatment (including the other treatment options available) and the benefits, risks and possible complications of the recommended treatment. This is essential so that the patient can make a decision. This is known as "informed consent". Once the information is given, the doctor may ask the patient to sign a consent form. When signed by the patient, this form gives the doctor legal permission to perform the procedure.

The consent form is generally seen to confirm the contractual agreement between the doctor and patient. Increasingly, this assumption has been challenged [6]. Legal and medical practitioners, as well as social researchers, have cogently argued that the complexity of language characteristic of consent forms, the anxious moment when the explanation is given, and the relatively short amount of time spent on explaining those aspects necessary to obtaining consent all render the consent form somewhat meaningless. Many consent forms seen or used show much use of legalistic and difficult language [6]. However, while the critics emphasize the complexity of the consent form, it should be emphasized as well that educational attainment level has a substantial impact on patient's ability to understand the consent form. Informed consent rests on four core principles which are: agreement, understanding, voluntariness, and the participant's right to withdraw at any stage of the process [7] but putting these elements into action is often challenging.

Consent is a process, not just a form. The process demands that information must be presented to enable individuals to voluntarily decide whether or not to accept treatment or participate in a research study. It ensures respect for people by providing the opportunity for thoughtful consent to ensure that participation is 
voluntary. In the broader literature [8-10] the key ethical issues discussed are informed consent, protection of participants, anonymity and confidentiality, and payment of a research participant. However the ethical issue of informed consent has generated the most debate globally with regard to healthcare needs and medical research.

Informed consent applies not only to the practice of medico-legal professionals but to the practice in all areas of medicine. Raab [11] said in many situations the informed consent process flows from a relationship between the doctor and patient, but when this does not occur, serious legal and ethical consequences may result.

\section{Law and Ethics}

For many years, common medical practice meant that medical care professionals make decisions for their patients. This paternalistic view has gradually been supplanted by promoting patient autonomy, whereby patients and physicians share the decision-making responsibility. Consequently doctor-patient relationships are quite different nowadays from they were two decades ago. However conflicts still abound as the medical community and those it serves struggle to define their respective roles in informed consent. The informed consent process as it currently exists often fails to accomplish its purposes [12]. Hallinan et al. [12] state that long and complex informed consent documents and processes may obscure the information most relevant to the potential patient and appear to be designed primarily to serve the role of protecting institutions and meeting regulatory needs rather than to inform the potential participant.

The law and ethics of informed consent both reflect and enforce the move from doctor-centered to patient-centered decision-making [10].

\section{Doctor-Patient Relationship}

The relationship is one of the categories of relationships protected by the equitable remedy against breach of confidence. Most courts that have considered the question have held that a physician who violates the physician-patient privilege is liable to the patient for damages. In PNG other healthcare workers such as nurses are trained in other special medical and administrative roles so that in the absence of a doctor or nurse could operate a health care facility. The difficulties may be too great but in principle the ethical standards should be the same for everyone. On this matter there are useful experiences to learn from PNG. Generally, many patients would respect the doctor, wait to see the doctor and majority would leave the doctor to decide what is best for them. The shift in doctor-patient relationship seems inevitable in hindsight in PNG.

In one consent case, a doctor told a woman he would only be repairing some cervical and rectal tears; instead he performed a hysterectomy [13]. If the patient was advised by her physician, the process nevertheless appeared so alien to the way the patient would normally make healthcare decisions. She did her best to understand the procedure she would undergo to the point where her comprehension failed and so her physician just has to decide for her. Acquisition of information relevant to decision-making is the first important step. The patient should be provided with all the information required, or additionally requested, to make a decision about undergoing the procedure. Information should be provided both verbally, visually or textually. In this case there were not many opportunities provided to the patient to consider and use them in the decision whether to consent or refuse the procedure. The patient did not understand the procedure and this in itself made her consent uninformed and thus she lacked any sense of autonomy.

Alternative grounds for legal proceedings do exist. Actions may be brought in contract and in negligence. In many instances, patients may be taken to have consented to certain disclosure of information, especially disclosure for purposes related to their own 
treatment, such as to other health service providers who assist their doctor to facilitate diagnosis but treatment of family members can not ordinarily be implied and will be likely to breach the duty of confidentiality, unless the disclosure is covered by some exception recognized by law. In other jurisdictions, the courts have extended this protection to other health care relationships, for example, nurse-patient. Negligence was held in a New Zealand case to be the basis on which an obligation of confidentiality [14] arose. There is no clear authority on this point in England and Wales in the medical context, although the potential for such an action can be seen by analogy to an action for negligent breach of disclosure of a police informer's identity [15]. In that case the principle of such an action was recognized although on its facts the case failed.

A quick examination of the laws in PNG, especially laws relating to health care, reveals that there are only a few laws which may be relevant to informed consent. It is unclear how much information each of the laws, rules or regulations offers on informed consent to medical treatment.

\section{Legal Basis of Informed Consent}

Consent is maybe the only principle that runs through all aspects of health care provisions today. It also represents the legal and ethical expression of the basic right to have one's autonomy and self-determination. If a medical practitioner attempts to treat a patient without valid consent then he or she will be liable under both tort and criminal law. Tort is a civil wrong for which the aggrieved party may seek compensation from the wrong doer. In certain extreme cases there is a theoretical possibility of criminal prosecution for assault or battery. The ethic governing health care practice is evolving rapidly, and individuals need to be sure that the principles of good practice are applied to all groups of patients. A major issue is the validity of consent.

Whatever might be the difference of approach it is evident that a medical practitioner is obligated to provide the necessary information before obtaining consent from a patient. To account for the PNG position, although we do not have much litigation, unlike in developed countries and other countries, it may be concluded that the courts have assigned immense significance to the requirement of informed consent. About the quantum of information to be given by medical practitioners, there are no clear parameters laid down by the courts, or even the Department of Health. Therefore it is reasonable information which a doctor deems fit considering best practices. Considering the high illiteracy in the country in this regard, the professional regulatory body for medicine can play an important role in establishing standards.

\section{PNG Law on Consent}

There are other legislations including: (i) Underlying Law Act 2000, (ii) Customs Recognition Act 1963, (iii) Medical \& Public Health Act, (iv) National Health Administration Act 1977, (v) Public Hospitals Act, and (vi) Provincial and District Health Boards Act, (vii) Workers Compensation (Miscellaneous Provisions) Act, (viii) PNG Criminal Code Act 1974, (ix) PNG Case Law, (x) other laws and government public policies that are relevant to informed consent in PNG.

While the law seems to acknowledge the existence of informed consent it does not often seem to clearly define it. In a list of various rights and freedoms, informed consent to some extent is implicit under section 49 of the Constitution. This section declares and guarantees every person the right to reasonable privacy in respect of their private and family life, communications with other persons and personal papers and effects, except to the extent that the exercise of that right is regulated or restricted by a law that complies with section 38 (general qualifications on qualified rights). We explain the application of section 38 immediately after the next paragraph which identifies and discusses the key components of the section 49 right to privacy. 
The key components of the right to privacy, for the present purposes, are reasonable privacy in relation to private and family life, communications with other persons and personal papers and effects. The privacy of an individual's life should cover their emotions, desires, priorities, and state of health, good or bad, and the freedom to disclose them to their family members or some other person. The individual's right of disclosure should remain at all times, except for a reasonably serious emergency, with the individual, and once a disclosure is made to a family member or someone else, say, a doctor, that ought not to be taken as a right to further disclosure. As well, a husband has no more right to delve into the health condition of the wife even if custom should dictate since the rules and principles of custom must comply with the written law, the Constitution being the supreme written law. In any case National Goal and Directive Principle 2 of the Constitution, even though non-justiciable, call for recognition of the principle that a complete relationship in marriage rests on equality of the partners' rights and duties. More importantly, section 55 of the Constitution declares that all citizens have the same rights, privileges, obligations and duties irrespective of race, tribe, and place of origin, political opinion, color, creed, religion or sex. It is self-evident that these rights and duties should extend to respect for the rights to privacy of each partner, including each one's confidential communication with a healthcare professional.

Such general qualifications under section 38 of the Constitution must take into account the National Goals and Directive Principles and the Basic Social Obligations which underlie the Constitution, be for the protection of the exercise of the rights and freedoms of others, and make reasonable provisions for cases where the exercise of one such right may conflict with the exercise of another, and the regulating or restricting law is reasonably justiciable in a democratic society having a proper respect for the rights and dignity of humankind. Obviously the right to privacy is a qualified right, as opposed to a fundamental right, such as the right to life, and therefore its exercise can be regulated or restricted in our jurisdiction. We have not found a judicial opinion that sheds light on the scope of the right to privacy nor is there an expert commentary on it.

Informed consent to medical treatment context has a different level of protection in PNG. Whilst it is unclear if the regulatory provisions relating to informed consent are established in PNG laws, the provisions basically are codification and extension of the Constitution, the courts, and the international standard and ethics on informed consent. In terms of health and medicals, it is unclear as to the extent to which they could offer protection. There have not been to date any legal cases where informed consent is the subject of judicial proceedings. There are no research studies that have examined the doctor-patient relationship. The legal system in PNG may be or is unaware of the country's situation and health care professionals' attitudes may go unchecked. The legal trend around the globe during the past two decades has been to increase the right of patients to greater freedom of choice. This fact has been insufficiently appreciated and has led to misunderstanding and confusion, further compounded by the courts' rhetoric that seems to promise more than it delivers. However failure to obtain informed consent to medical treatment renders the medical care professional(s) liable for lack of consent, negligence or battery or assault, and constitutes medical malpractice.

A young boy's circumcision is an important rite of passage for the young male person to manhood in Manus Province [16] of PNG. The village elders perform the procedure in a male sacred place. In a few cases young people have contracted herpes as a result of the procedure and brought to hospital. People still adhere to their customary beliefs and norms, and practice them. The young man in this case may be unaware of the procedure he has to get through, which is a customary requirement and which he obeyed and 
followed gave his consent uninformed and lacking any sense of autonomy. Just as in many indigenous communities in PNG, a young person expects his or her family to make a decision for him or her.

The lack of efforts into developing topic has far-reaching consequences, pushing the need for development of consent law to new heights. It is contended that it is not only informed consent which is imperative now, but the same shall apply to "prior informed consent" unless there is an imminent threat to the life of the young man of the preceding story. In addition this decision curtails the scope of proxy consent from a person whose authority comes from his parents or the attendant. Interestingly, in PNG, the entire gamut of laws on consent turn into a complex proposition if an emergency medical situation arises.

\section{Discussion and Medical Understanding of Informed Consent}

We start this discussion by mentioning several different views within the medical community regarding informed consent. Then we discuss the criticisms of the idea of informed consent as well as several suggested improvements to the process. We continue to discuss how other obstacles can impact the process of obtaining informed consent. We wrap up by discussing ways to improve patient decision-making based on informed consent.

We say that within certain social and economic positions in a society, the issues of patients being misinformed can be more or far less prevalent. Some ideas may not translate well across language barriers or other ideas may seem more offensive within certain cultural boundaries. In other traditional settings where inhibiting customs are widespread, patients may refuse to make a medical decision for their health care needs. A patient may have religious or cultural beliefs or opinions which would affect his or her own personal decisions, and though they may not be supported by scientific evidence, they are still completely relevant to the patient's final decision, and so the information given should be relevant to that. The need for an important doctor-patient relationship and discussion is clear, as without an understanding of the patient's beliefs, a doctor may completely overlook relevant information and fail to achieve valid informed consent [10].

In PNG indigenous local customs could influence judgements about the amount and type of information that should be disclosed to the patients by healthcare professionals for informed consent. Traditional customs, languages and other social factors could affect the way of life of the people. In many communities, the situation regarding medical informed consent to treatment is unknown and thus more research work is required in this area. As a result it is further unknown whether or not the rights to making decisions for healthcare needs have or are being interfered with by factors including particularly customs, languages, high illiteracy rate in the communities, and hence further leading to poor health status of women, geriatric population, and young adults.

A physician's responsibilities are clearly informed in the practice code of conduct. Patients may accept or refuse treatment based on their customs, beliefs, or opinions. The relevant stakeholder groups in law and medical practice, academics, industry, government, associations, community leaders, patients and consumer interest groups should work together to deal with the circumstances where the issue lies especially with the patient's custom taking a more determinant role in their health care than the patient would otherwise like. However there is not much a provider of health care can do without violating certain patients' rights. The sensitivity of the topic however requires that the issue should be addressed with great care. There exists a vast discrepancy between what informed consent ought to be and what patients perceive it to be.

For most medical settings, especially in developed countries, informed consent is documented using a written document, the "consent form", which provides 
information regarding the treatment or medical procedure. Informed consent form is intended, in part, to provide information for the potential subject's current and future reference and to document the interaction between the subject and the medical caregiver. However, even if a signed consent form is required, it alone does not constitute an adequate consent process. Informed consent process is an ongoing exchange of information between the physician and the subject and could include, for example, use of question-and-answer sessions, community meetings, and videotaped presentations.

The health caregiver professional ought to understand the impact of traditional customs of the people on their basic values, priorities, norms and beliefs, opinions, and behaviors. These are essential requirements that should be understood to truly attain people's trust and confidence. Marshall [17] points to the need for detailed research of the socio-cultural and customary context of the communities in the developing countries.

\section{Obstacles to Informed Consent}

\subsection{Comprehension and Capacity}

Although language is an issue in securing informed consent, obstacles to comprehension go beyond the obvious linguistic barriers. The intervention of an interpreter may help but different concepts of illness and issues of translation and cultural bias on the interpreter's part would compromise the extent to which information is understood.

PNG is one of the most heterogeneous countries in the world, having over 800 indigenous tribes and languages where recognition of individual rights is poor and the divide in language, customs and traditions has led to many different groupings in the community. Among the cultural groups there may be little or no understanding of biomedicine and healthcare workers, lacking knowledge of traditional belief systems, may wrongly conclude that the individual lacks capacity.

\subsection{Literacy}

Many participants in the study are from different parts of the country. Majority of the patients are unable to read and write in their own languages. The communication amongst the population is largely verbal and is heavily relied upon. To ask for a signature may thus cause disorientation of the patient. Illiteracy does not mean the information is misunderstood but it does mean the information must be presented in another or a special way. Obtaining patient's informed consent in this case must be seen to be a continuing process of negotiation between the healthcare professional and the patient. This implies a long-term relationship of trust.

\subsection{Autonomy}

The notion of autonomy varies considerably between cultural groups. In contrast to the emphasis on personal choices in Western countries including USA and Europe, communal and hierarchical concepts of decision-making take precedence amongst the indigenous populations mostly in developing countries. The family members, even clan elders will frequently participate in making decisions on many issues including medical for and on behalf of a relative. The sense of wellbeing depends on a feeling of personal control [18]. Dein and Kamaldeep [19] expressed that the Western idea of respect for the individual may conflict with traditions that define persons by their relations to others. In the doctor-patient relationship elements such as loyalty, integrity, solidarity and compassion may be more important in situations such as this among the indigenous populations and minority groups, than autonomy.

\subsection{Disclosure of Information}

The patients must have adequate information if they are to play an important role in making decisions that reflect their own values and preferences, and the physicians play a key role as educators in this process. Treatment with no consent at all, actual or implied, 
treatment substantially different from that to which the patient consented, or unauthorized substitution of one treater for another come within the definition of battery, especially when involving invasive procedures. An unpermitted medical treatment may be lifesaving or curative, but except in situations where consent would be implied, does not excuse battery [20]. For example, the courts in Mohr's case recognized a cause of action for battery when medical treatment was provided without the patient's consent [21].

The extent of information that is disclosed to individuals at the clinical settings varies due to circumstances in which the individuals operate and live that influence their health care outcome needs. For example, when a life-threatening condition such as cancer is diagnosed, the custom in many parts of the world is to tell the family relatives rather than the patient. This is generally true of South East Asian cultures [19] and Pacific Islands. Among such groups the disclosure of negative information is considered potentially harmful [19], and health personnel will sometimes collude with the family relatives to prevent the patient discovering the diagnosis [22]. Diversity in the practice of disclosing information would alter the requirements for informed consent in the medical context [23].

\subsection{Familiarity with Conduct of Research}

Patients and groups living in rural areas and isolated from the mainstream society have little grasp of scientific method, and without such understanding a consent form will make little sense. This may be partly a cultural issue, though low literacy amongst the people in the communities is also an important determinant. Work in countries in South Asia, including India, Pakistani and Bangladesh, showed no difference from the general population in their attitudes toward participating in clinical trials [24].

There is no difficulty with informed consent in any context, especially when the researcher is an outsider and the potential subjects are patients and healthcare workers. It was an unequal power relationship and yet there was general feeling of obligation and support for this study. Where an asymmetry in knowledge and authority occurs, and we have not confronted such a case, this must raise doubts about the validity of consent [25].

\subsection{Misconceptions}

There is a great deal of misconception towards legal status of informed consent. In many developing countries majority of the people live in their indigenous settings, trust their doctors to do the right thing and do not mind after saying "yes” to a medical procedure and they are made better. Patients believe that they have no right to change their mind after saying “yes” to undergo a medical procedure. The level of understanding of health care seems low among indigenous populations and issues of custom and culture have bearing on the patients' decisions for health care [26]. A small group of Christians believe in faith healing and use prayer and other religious activity rather than medicine to take care of complicated medical difficulties [27]. Some of those patients may have died of treatable diseases like childhood diabetes, measles and diarrhea after medical care was not taken. Cases such as this should have resulted in criminal charges or convictions for those responsible [27]. Religious needs, regardless of whether or not an institution accommodates for those needs, can often come into conflict with the western ideals for medicine.

Any attempt by healthcare workers to explain the issues would cover scientific research and how it works but there would be difficulty in addressing a tradition of healthcare in which spiritual and religious values and a host of different healing methods are deployed alongside conventional medicine. For instance, in an informed consent study in settings of African descent, researchers compared voluntary participation and understanding of informed consent among the individuals with hypertension living in United States and Nigeria [23]. Survey questionnaires were used to 
evaluate factors associated with voluntariness and understanding of the study's purpose. It found that there was a need for more effective approaches and interventions to improve comprehension of consent in genetic research among ethically and linguistically diverse populations.

The process of receiving healthcare, however, involves the sharing of information and thus potentially threatens individual control over privacy. When patients seek treatment, they give health professionals (e.g., doctors, nurses, and others) access to their bodies, provide information about their habits and personal relationships and provide access to their otherwise private world. It is through the intrusion of medical tests results, records of which and other known information about the patient are kept before they do. This implies a duty of confidentiality. Thus the professional duty of confidentiality arises because of the loss of privacy in that it refers to the duty of doctors not to disclose to others the information that a patient divulges and the corresponding right of patients not to have the information they divulge made available to anyone else [28].

Several courts have concluded that public policy favors the protection of the confidentiality-relationship between a doctor and patient [21]. In courts in U.S., they found evidence of this public policy in state testimonial privileges, unprofessional conduct statutes, physician licensing statutes, and statutes limiting the availability of medical records [21]. Other courts have applied such statutes more directly, finding that a physician who breaches such standards by improperly disclosing medical records of information may be liable for breach of contract to the patient. The existence of this broad general principle reflects the fact that there is such a public interest in the maintenance of confidentiality that the law will provide remedies for this protection. The relationship is one of the categories of relationships protected by the equitable remedy for breach of confidence.
Now the question to ask is, can the notion of informed consent be properly applied in PNG to the indigenous populations who abide by their traditions, customs, beliefs and who follow strongly their views and opinions?

\section{The Impact of Cultural and Religious Milieus}

One important area where questions still arise with regards to informed consent is the peoples' customs, cultures and languages. There are factors between peoples of differing circumstances which can greatly alter how they view consent. Some groups would involve people in the decision-making process that may not traditionally be involved in the decision making of a medical decision such as in the case of Japanese traditional culture [29]. Other groups may dislike certain medical procedures as in PNG. And certain people have different views on what should be disclosed of the patient's condition.

Customs are common phenomena which continue to affect the daily lives of many thousands of people. It is unclear in PNG about the characteristics of customs on health care because there are no published information on informed consent and issues that affect the making of informed consent. A patient should be informed of anything which would affect his or her personal decisions, such as anything which would upset any belief the patient has, particularly among female patients where rules of custom are adhered to in the community [30]. Where customs, beliefs, and opinions of individuals are taken into account in informed consent, patients make better considered decisions which affect their health care. Beliefs associated with illness experiences are embedded in customs and cultural values that may have implications for the implementation of informed consent processes [23]. The differences in these values could influence judgements about the amount and kind of information that should be disclosed by healthcare professionals to the patients. The health care professionals may 
know ways of treating the patient-doctor relationship. In situations such as dealing with women patients, usually the physician and patient participate in the process of deciding what the best mode of treatment is for the patient. Unless the patient willingly forgoes her decision-making power and leaves her fate in another's hands, then the doctor must work at creating an atmosphere conducive to the patient's understanding and response to the various treatment options.

There are also inequities within the population groups because of high unemployment and low literacy in the community. The basic health care is unaffordable or out of reach for most of the local population, therefore the majority still patronizes traditional healers for their health care needs. Under these circumstances any offer of medical assistance is often seen as better than nothing, thus encouraging undue influence, coercion and medical paternalism. Perhaps there is a further dichotomy in the organization of the healthcare services in PNG, which is dual in nature, consisting of public health care and private medical service. The private service is patronized by a small portion of the population who can afford private health care insurance or possess the financial means to pay for the private care.

The need for an important doctor-patient relationship and discussion is clear, as without an understanding of the patient's beliefs, a doctor may completely overlook relevant information and fail to bring about a valid informed consent. It is necessary to understand the characteristics of customs, and peoples' habits and practices. Traditional and spiritual healers attend to many patients from poor socio-economic standing and patients from remote rural areas who can not afford to travel long distances into urban areas for their health needs. A healer uses herbs, medicinal plants, sorcery and attends to patients as if in a medical setting. In many communities, traditional healers are among the traditional leaders, on par with the village chiefs, village headmen and spiritual leaders [31].
They are custodians and enforcers of traditional customs in the community. It is easier for people to respect instructions that contradict their own values when they are given by well-respected leaders as compared to unfamiliar health workers. However as evidenced in some of the communities, misinformed traditional leaders can hinder preventive efforts. On the other hand, well informed traditional leaders can potentially play crucial roles in reducing the health problems. Traditional healers help with the patients although the practice by a healer is not acceptable and the government refuses to recognize that practice.

The aspect of religion apart from culture, also can not be ignored as many religious groups, for example Jehovah's Witnesses, take issue with several medical procedures that hospitals are required to perform when quick decisions are needed and consent may not be obtained as time is short and decision is needed to save the patient. Jehovah's Witnesses believe that God forbids the consumption of blood, and this includes transfusions [32]. Some bypass this religious prohibition by withdrawing their own blood prior to an operation, but this is not always the available option during emergency operations. In some cases, parents refuse to allow their children to have blood transfusions, even in life-threatening situations leading to death of the children. Hospitals in the past typically approached child-welfare authorities who asked the courts for an order giving them temporary custody so they can ensure the transfusion is administered [32]. Moreover, some Jehovah's Witnesses have relaxed their stance on blood transfusion [32].

The perception all over the world is that these factors (indigenous customary procedures) have a big impact on the decision making for medical treatment. The degree to which we should respect, or challenge such behaviors, customary rules and practices, is a recurring tension in the multicultural and contemporary health care context. The peculiarities of customs of indigenous people may explain some of the actions relevant to informed consent. 


\section{Preserving Community Norms and Family Relationships}

In a study among the African Americans, the participants felt as though by signing consent forms, healthcare workers were gaining some legal protection while they themselves were signing away their autonomy [33]. Emmanuel and Joffe [34] said that while the model of signing informed consent form may fit comfortably with most Western societies, some cultures may not consider informed consent to be a universal ideal, and preserving community norms and family relationships is more important than individual autonomy. The following examples explore this circumstance.

In PNG, a young patient's choice over medical treatment may be in opposition to the preference of the parents and older family members, and sometimes an extended family decision must be considered. Among the same group of people, married women may not be deemed capable of making medical decisions for them instead that right is given to their husbands [23]. Similarly in Africa, most women can not go to family planning clinics without their husbands' approval or even if they attend, the right to receive family planning method requires the husband to give his decision [23]. In other societies, women may not be deemed capable of making medical decisions and so their rights are given to their husbands. For example, in Arab culture, decisions are made at the collective, family, tribal, or community level, not by an individual [13]. Consequently, female patients had little need for extensive explanation of the risks and benefits of the proposed medical treatment. Generally, in many indigenous settings, the right of informed consent is transferred to the immediate family and the extended family relatives and thus this cultivates an idea that the patient takes a passive role in his or her healthcare which is in great contrast to the Western ideas of informed consent.

The most important goal of informed consent is that the patient should have an opportunity to be an informed participant in his or her healthcare decisions to safeguard and ensure the preservation of individual rights. The pragmatic suggestions aim to facilitate and document a good-faith effort to involve patients in medical decisions to whatever degree they are interested and able. Such practice complies with the ethical spirit of informed consent and should minimize legal conflict by fostering a deep and nuanced respect for patients.

\section{Conclusion}

Most people would have been assisted at a health care center by healthcare professionals as physicians, nurses, and other healthcare workers. Submission to treatment justifiably implies an understanding of the procedure, and willingness to undergo it. Resort to law is confined to the most egregious cases in which healthcare professionals visibly harm the patients by doing something that the patients clearly did not desire or foresee, for example the removal of a kidney and not the appendix which is cause of the complaint.

There is lack of information dealing with policy guidelines regarding physician-patient relationship which hinders growth in the health care industry. Health policies are geared to improving approaches to the delivery of health care. Health care law as a backdrop of medical practice has an impact on the people and the communities. The law plays a role that runs wider and deeper than addressing patient grievances and misconduct of a health care worker. While health worker's responsibilities are clearly outlined in their code of practice, customs, cultural beliefs and opinions prevail among the population who may accept or refuse treatment based on their personal opinions or beliefs. The vulnerable populations including children, pregnant and lactating mothers, and prisoners must receive extra protection.

One critical issue which needs to be examined in light of the effects on informed consent is innovative and culturally responsive ways to disclose information to individuals as patients. 


\section{References}

[1] Douglas, G. Andrew. "Informed Consent for All! No Exceptions”. 37 N.M.L. Rev. 39 (2007). Available at: https://digitalrepository.unm.edu/nmlr/vol37/iss $1 / 4$.

[2] The National Commission for the Protection of Human Subjects of Biomedical and Behavioural Research. 1997. The Belmont Report Ethical Principles and Guidelines for the Protection of Human Subjects of Research. http://www.ohsr.od.nih.gov/guidelines/belmont.html.

[3] Faden, R. R., and Beauchamp, T. L. 1986. A History and Theory of Informed Consent. New York: Oxford University Press, 1-392.

[4] Gel Del, C. M., and Joffe, S. 2005. "Informed Consent for Medical Treatment and Research: A Review.” The Oncologist Medical Ethics 10: 636-41.

[5] Spencer, C. V. 1972. DC Cir 464 F2d 772.

[6] Law Reform Commission of Victoria. 1987. Informed Consent to Medical Treatment. Discussion Paper No. 7, Melbourne: Law Reform Commission.

[7] Parmar, P., Gunvanti B. R., Sangita, R., and Ashish, P. 2016. "Consent in Medical Practice-Perceptions of Patients towards Legal Aspects of Informed Consent”. International Archives of Integrated Medicine 3 (4): 105-110.

[8] O’Neill, O. 2003. “Some Limits of Informed Consent.” $J$ Med Ethics 29: 4-7.

[9] Hall, D. E., Prochazka, A. V., Frank, A. S. 2012. "Informed Consent for Clinical Treatment." Canadian Medical Association Journal 184 (5). doi:10.1503/cmaj.112120.

[10] Allmark, P., et al. 2010. "Ethical Issues in the Use of In-Depth Interviews: Literature Review and Discussion.” Sheffield Hallam University Research Archive (SHURA) http://shura.ac.uk/1372/.

[11] Raab, E. L. 2004. "The Parameters of Informed Consent.” Trans Am Ophthalmology Soc 102.

[12] Hallinan, Z. P., Forrest, A., Uhlenbrauck, G., Young, S., and McKinney Jr., R. 2016. "Barriers to Change in the Informed Consent Process: A Systematic Literature Review.” IRB: Ethics \& Human Research 38 (3): 1-10. http://thehastingscenter.org/for-media/.

[13] Berg, J. W., Applebaum, P. S., Lidz, C. W., et al. 2001. Informed Consent: Legal Theory and Clinical Practice. Oxford: Oxford University Press, 1-340.

[14] “Furniss v Fitchett (1958). N.Z.L.R 396.”

[15] The NZ Times, May 25, 1999.

[16] Personal Communication with the Village Elders, Loss Negros Island, Manus Province, PNG, December 2018.

[17] Marshall, P. A. 2007. Ethical Challenges in Study Design and Informed Consent for Health Research in Resource-Poor Settings. Geneva, Switzerland:
TDR/World Health Organization.

[18] Saxena, S. 1994. "Quality of Life Assessment in Cancer Patients in India.” In Cross Cultural Issues in Quality Life Assessment: International Perspectives, edited by Orley, J., Kuykyn, W., Berlin: Springer-Verlag, 99-107.

[19] Dein, S., and Kamaldeep, B. 2005. "Issues Concerning Informed Consent for Medical Research among Non-westernized Ethnic Minority Patients in the UK." Journal of the Royal Society of Medicine 98: 354-6.

[20] 1905. Mohr v William. Minn. 261, 104 N.W. 12.

[21] Sugarman, J. 2015. "Informed Consent: Why Do We Care?” In Informed Consent and Health Literacy: Workshop Summary. Washington D.C.: The National Academies Press. www.nap.edu.

[22] Chaturverdi, S. 1994. "Exploration of Concerns and Role of Psychosocial Interventions in Palliative Care-A Study from India.” Ann Acad Med Singapore 23: 256-60.

[23] Marshall, P. A., Adebamowo, C. A., Adyemo, A. A., Ogundiran, O. T., Vekich, M., Strenshi, T., Jie Zhou, B. S., Prewitt, E. T., Cooper, S. R., and Rotimi, N. C. 2006. "Voluntary Participation and Informed Consent to International Genetic Research.” American Journal of Public Health 96 (11).

[24] Husain-Gambles, M., Leese, B., Atkin Brown, J., Masson, S., and Tovey, P. 2004. "Involving South Asian Patients in Clinical Trials.” Health Technol Assess 8: 73-9.

[25] Murray, T. 1994. "Individualism and Community: The Contested Terrain of Autonomy." Hastings Center Rep. 24: 32-5.

[26] Joseph, M., Francis, M., Malcolm, M., Paul, N., and Abdullah, C. 2008. "Why Do People Refuse to Take Part in Biomedical Research Studies? Evidence from a Resource-Poor Area.” Malawi Medical Journal 20 (2): 57-63.

[27] "Time: When Parents Call God Instead of the Doctor." http://.www.time.com/time/nation/article/0.8599.1877352 .00.html.

[28] Richards, B., and Louise, J. 2014. Medical Law and Ethics. A Problem-Based Approach. Australia: LeisNexis Butterworths.

[29] Saldov, M., Kakai, H., McLaughlin, L., and Thomas, A. 1998. "Cultural Barriers in Oncology: Issues Obtaining Medical Informed Consent from Japanese-American Elders in Hawaii.” Journal of Cross-Cultural Gerontology $13 . \quad$ Retrieved from http://download.springer.com/static/pdf/740/art\%253A10. 1023\%252FA\%253A1006532713142.pdf?auth66=14188 45096_8cecc436ef33954bce47e27581bbe90d3\&ext=.pdf.

[30] Culver, C. M., and Gert, B. 1982. Philosophy in Medicine: Conceptual and Ethical Issues in Medicine and Psychiatry. New York: Oxford University Press.

[31] Manguvo, A., and Mufuvadze, B. 2015. “The Impact of 
Traditional and Religious Practices on the Spread of Ebola in West Africa: Time for a Strategic Shift.” The Pan African Medical Journal 22 (Suppl 1): 9. doi:11694/pamj.supp.2015, 22.1.6190.

[32] National Post: Without Fanfare. Jehovah's Witnesses Quietly Soften Position on Blood Transfusions http://life.nationalpost.com./2012/12/20/without-fanfare-j ehovahs-witnesses-quietly-soften-position-on-blood-trans fusions/.
[33] McGabe, M., Morgan, F., Curley, H., Begay, R., and Ghodes, D. M. 2005. "The Informed Consent Process in a Cross-Cultural Setting: Is the Process Achieving the Intended Result?” Ethnicity \& Disease 15. Retrieved from http://www.t.ishib.org/journal/ethn-15-2-300.pdf.

[34] Emmanuel, E. J., and Joffe, S. 2000. "Ethics in Oncology." In Cancer Medicine, 5th ed., edited by Bast, R. C., Kufe, D. W., Pollock, R. E., et al., Hamilton: B.C. Decker, Inc., 1145-63. 